\title{
Microdrop Deposition Technique: Preparation and Characterization of Diluted Suspended Particulate Samples
}

\author{
Salvatore Macis 1,2,*, Giannantonio Cibin ${ }^{3}$, Valter Maggi ${ }^{4,5}$, Giovanni Baccolo ${ }^{4,5}$ (D), \\ Dariush Hampai $^{2}$ (D), Barbara Delmonte ${ }^{4}$, Alessandro D'Elia ${ }^{6}$ (D) and Augusto Marcelli ${ }^{2,7}$ (iD \\ 1 Department of Mathematics and Physics, Università di Roma Tor Vergata, via della Ricerca Scientifica 1, \\ 00133 Rome, Italy \\ 2 Laboratori Nazionali di Frascati, Istituto Nazionale di Fisica Nucleare, 00044 Frascati, Italy; \\ dariush.hampai@lnf.infn.it (D.H.); augusto.marcelli@lnf.infn.it (A.M.) \\ 3 Diamond Light Source, Harwell Science and Innovation Campus, Didcot OX11 0DE, UK; \\ giannantonio.cibin@diamond.ac.uk \\ 4 Dipartimento di Scienze dell'Ambiente e della Terra, Università degli Studi di Milano Bicocca, Piazza della \\ Scienza, 1-20126 Milano, Italy; valtermaggi@gmail.com (V.M.); giovanni.baccolo@mib.infn.it (G.B.); \\ barbara.delmonte@unimib.it (B.D.) \\ 5 Sezione di Milano-Bicocca, Istituto Nazionale di Fisica Nucleare, Piazza della Scienza, 3-20126 Milano, Italy \\ 6 Department of Physics, University of Trieste, Via A. Valerio 2, 34127 Trieste, Italy; ale9149@gmail.com \\ 7 Istituto Officina dei Materiali, Consiglio Nazionale delle Ricerche, Basovizza SS-14, km 163.5, \\ 34149 Trieste, Italy \\ * Correspondence: salvatore.macis@roma2.infn.it; Tel.: +39-067-259-4523
}

Received: 23 May 2018; Accepted: 11 July 2018; Published: 16 July 2018

\begin{abstract}
The analysis of particulate matter (PM) in dilute solutions is an important target for environmental, geochemical, and biochemical research. Here, we show how microdrop technology may allow the control, through the evaporation of small droplets, of the deposition of insoluble materials dispersed in a solution on a well-defined area with a specific spatial pattern. Using this technology, the superficial density of the deposited solute can be accurately controlled. In particular, it becomes possible to deposit an extremely reduced amount of insoluble material, in the order of few $\mu \mathrm{g}$ on a confined area, thus allowing a relatively high superficial density to be reached within a limited time. In this work, we quantitatively compare the microdrop technique for the preparation of particulate matter samples with the classical filtering technique. After having been optimized, the microdrop technique allows obtaining a more homogeneous deposition and may limit the sample amount up to a factor 25 . This method is potentially suitable for many novel applications in different scientific fields such as demanding spectroscopic studies looking at the mineral fraction contained in ice cores or to pollution investigations looking at the detection of heavy metals present in ultra-trace in water.
\end{abstract}

Keywords: ultra-dilution; droplets; water; evaporation; X-ray fluorescence

\section{Introduction}

When dealing with complex samples, one of the main bottlenecks from the practical point of view is their preparation. A suitable sample preparation method should allow saving time during data acquisition, enhancing the $\mathrm{S} / \mathrm{N}$ ratio, improving the detection limits, etc. Considering particulate matter (PM) analysis of solutions, the most common preparation technique is filtration: it is easy, reliable, and fast, but at the same time, it presents some drawbacks. Indeed, it requires a considerable 
sample consumption and PM whose size is smaller than the one of the filtration pores, which are not retained on the filter. To avoid this point, the original solution could be deposited on a membrane presenting a quite large surface (about $1 \mathrm{~cm}^{2}$ ). In this case a critical issue is the low superficial density of the PM deposited on top of it.

The evaporation process, based on the deposition of micro-droplets, may overcome both issues. Indeed, the microdrop technique consists in the deposition of PM in association to liquid drops presenting a diameter between 70 and $200 \mu \mathrm{m}$. After the evaporation of the solvent, the PM depositional pattern resembles the one of the original droplets. The main disadvantages of this method are the inhomogeneity of the deposition and the long time required for the evaporation of the solvent. In fact, the evaporation of large drops (few $\mathrm{mm}$ of diameter) can last several hours. At normal conditions of temperature and humidity, full evaporation of drops of water can require more than $10 \mathrm{~h}$ per $\mathrm{mL}$, and the density fluctuation of the deposited material is up to $80 \%$ as a consequence of the so-called coffee-stain effect [1]. To optimize the process, we deposit micro-droplets and control the deposition area with motorized translational stages. To this purpose, a study of the evaporation dynamics is necessary to investigate the uniformity of deposition and to evaluate the evaporation rate of micro-droplets presenting different sizes. When dealing with densely-packed deposition patterns, our results show that is necessary to consider the interaction between different evaporating droplets.

The evaporation of a drop on a surface can be described with two different models: the evaporation with a "constant contact area" and that with a "constant contact angle" [2] (see Figure 1).
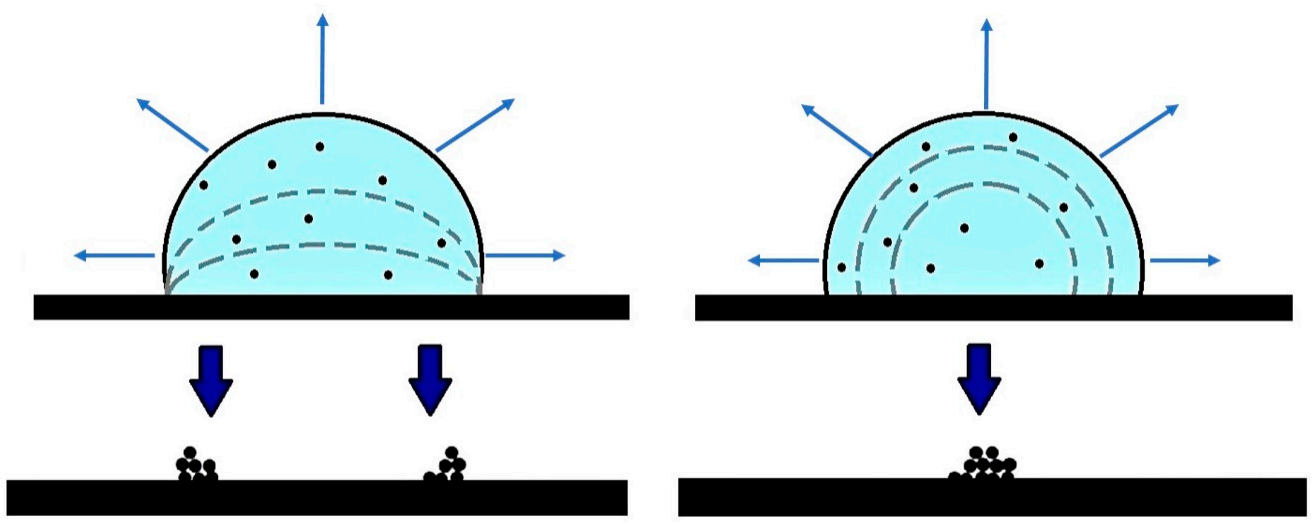

Figure 1. Deposition and evaporation processes for two different models: "constant contact area" (left) and "constant contact angle" (right). The main difference is represented by the shape of materials deposited on the substrate: pinned at the edge for the "constant contact area" model (bottom left) and concentrated in the center for the "constant contact angle" model (bottom right).

In the first case, the evaporation takes place, maintaining a constant contact area between the liquid drop and the surface. The shape of the drop remains almost "spherical" [2], but the contact angle decreases. In accordance to the second model, the contact angle of the edge of the drop is constant during the evaporation [2], thus, the shape of the drop remains "spherical", but the contact area between liquid and surface continuously decreases. The "constant contact area model" is suitable when a strong interaction between the liquid and the substrate takes place, e.g., when considering water and a hydrophilic surface [3,4]. This is clear in the work of Peschel et al., where at higher concentration, the evaporation occurs with the constant area mode, i.e., during the evaporation, the droplet does not shrink [5].

On the opposite, the second model is more appropriate when the drop-substrate interactions are weak, as in the case of water on a hydrophobic substrate. As studied by Fittschen et al. [6], an ultra-diluted solution is characterized by a low solution-substrate interaction, and the coffee stain phenomenon is reduced. As a matter of fact, the insoluble components deposed by the evaporation of the droplets have to be characterized in order to achieve the most homogeneous deposition starting 
from defined droplet size. As shown by Sparks et al., droplet dimension and solution concentration are the main parameters that control the deposition [7]. Thus, the deposition method we presented can be a fast, simple, and direct way to achieve a homogeneous deposition of insoluble materials considering only the droplet size and the concentration of the solution.

In this contribution, we will describe the optimization process of an experimental setup based on the evaporation of water micro-droplets in accordance to the constant angle model. The uniformity of the deposition will be determined, and results will be compared to those obtained through the standard filtration method. Indeed, the microdrop method may offer many opportunities and dedicated preparations in different scientific fields. One particular demanding application is the homogeneous deposition onto specific substrates of inorganic components, e.g., coarse particles, mineral fractions, and particulate matter for spectroscopic characterizations. Research that could benefit from this particular type of sample preparation are ice core studies or detection of heavy metals present in ultra-trace elements.

\section{Experimental}

Using a micro-dispenser, micro-sized droplets can be deposited, controlling the spatial distribution of drops, their size, and the deposition rate. The Microdrop technology [8] allows spreading of extremely small amounts of a liquid solution. Moreover, thanks to dedicated devices, it is possible to deposit single droplets whose volume is in the sub-nanoliter range. Such small droplets present an interaction with the substrate, which can be considered negligible. The micro-dispenser is composed of a head with a nozzle and a pumping chamber working with the same piezoelectric technology used for inkjet printers. The head is controlled by a driver, i.e., a pulse generator, which controls the piezoelectric actuator and sends pulses to the head. The device we used is the MD-K-130 (C) Microdrop Technologies $\mathrm{GmbH}$, Norderstedt, Germany). With an inner nozzle diameter of $70 \mu \mathrm{m}$, we may produce, with $1 \%$ volume repeatability, droplets with a minimum nominal volume of $180 \mathrm{pl}$ and at a drop rate from 1 to $2000 \mathrm{~Hz}[8,9]$. To prepare a sample with a specific microdrop pattern, it is necessary to control the number of drops on the substrate, releasing each of them at a well-defined and repeatable distance. In our experimental setup, to generate the pattern, the head is maintained fixed while the substrate translates under the stream of droplets released by the head. The motion of the substrate is realized with two precision PI Micos (Physik Instrumente GmbH Karlsruhe, Karlsruhe, Germany) Translational Stage VT-80 stages, assembled perpendicularly with respect to each other. This setting allows positioning the substrate with a nominal accuracy of $1 \mu \mathrm{m}$ per axis. The desired spatial patterns are obtained controlling the two translation stages with a LabView@-based code and properly setting the Microdrop dispenser [9].

The characterization of the evaporation time and of the drop pattern was made with the Zeiss Axio Imager M1 optical microscope (Carl Zeiss Microscopy LLC, Oberkochen, Germany) using 5×, $10 \times$, and $20 \times$ magnification optics. The video camera had a $x-y$ spatial resolution of $0.13 \mu \mathrm{m}$ at $5 \times$, $0.26 \mu \mathrm{m}$ at $10 \times$, and $0.52 \mu \mathrm{m}$ at $20 \times$ magnification and allowed us to measure the droplet volume with an uncertainty of $\sim 3 \%$ of the measured volume. We used a solution of ink and water (Gullor ${ }^{\circledR}$ ink diluted at 50\% with high purity milliQ water) for the optical analysis of the deposition, and high purity milliQ water for the evaporation dynamic measurements, i.e., the diameter of the drop and the angle of contact. The substrates we used for the evaporation analysis were Kapton ${ }^{\circledR}$ polyimide films from DuPont of $0.005^{\prime}(125 \mu \mathrm{m})$ thickness, while we used polycarbonate Nucleopore $\bigodot$ membrane filters (pore size $0.45 \mu \mathrm{m}$ and filter area diameter $\sim 20 \mathrm{~mm}$ ) for the analysis of morphology on evaporated samples morphology by X-Ray Fluorescence.

The evaluation of the PM deposition uniformity has been performed using the X-ray fluorescence technique at the beamline B18 at the Diamond Light Source facility (Harwell, United Kingdom) [10], using a monochromatic X-ray beam at $8 \mathrm{keV}$, with a focus of $100 \mu \mathrm{m}$ radius and a detection limit of $100 \mathrm{ng} / \mathrm{mm}^{2}$. 
The evaluation of the evaporation was carried out measuring the diameter and height of droplets as a function of time using the optical microscope and the camera. We measured the parameters of the single droplets and of groups of droplets to evaluate the evaporation rate as a function of the size and of the distance between several droplets. We then identified the minimum deposited area achievable with the evaporation of small droplets, in the shortest time. To optimize the deposition process, all images and experimental parameters were analyzed and used to write an approximate model.

\section{Results}

The analysis of the images of the droplets (see Figure 2) and of the measured parameters clearly points out that when using a low concentration solution and a hydrophobic substrate such as Kapton, the evaporation occurs in accordance to the "constant angle" model, well described by the theoretical model introduced by Picknett and Bexon in 1977 [2]. The result is confirmed in Figure 3, where our data are compared to the "constant angle" and "constant contact area" models. The deposition rate, i.e., the volume of the liquid deposited vs. time, was optimized considering the velocity of the stages and the spatial pattern parameters of the deposited droplets. Indeed, a fundamental parameter that drives the evaporation is the saturation of the atmosphere surrounding the droplets' selves, which is directly related to the mutual position of the droplets. Different droplet sizes and different distances between successive droplets were considered to this aim. In Figure 4, we show the correlated parameters of the deposition tests we performed using the translation stages combined with the microdrop. We defined the area to cover (simulating an experimental condition, e.g., the deposition of $1 \mathrm{~mL}$ of solution onto an area of $\sim 35 \mathrm{~mm}^{2}$ ) and then showed data using the distance between two consecutive drops along the deposition line, and the diameter of the droplet to be deposed as the two axes. The third axis represents the evaporation rate density, i.e., the amount that can be deposed on the surface area (defined above) per unit/area. Data presented in Figure 4 point out that the highest evaporation rate at $20{ }^{\circ} \mathrm{C}\left(\sim 0.7 \mathrm{~mL} / \mathrm{h} \mathrm{cm}^{2}\right)$ is obtained setting the droplet diameter at $240 \mu \mathrm{m}$ and the distance between two consecutive droplets at $170 \mu \mathrm{m}$.

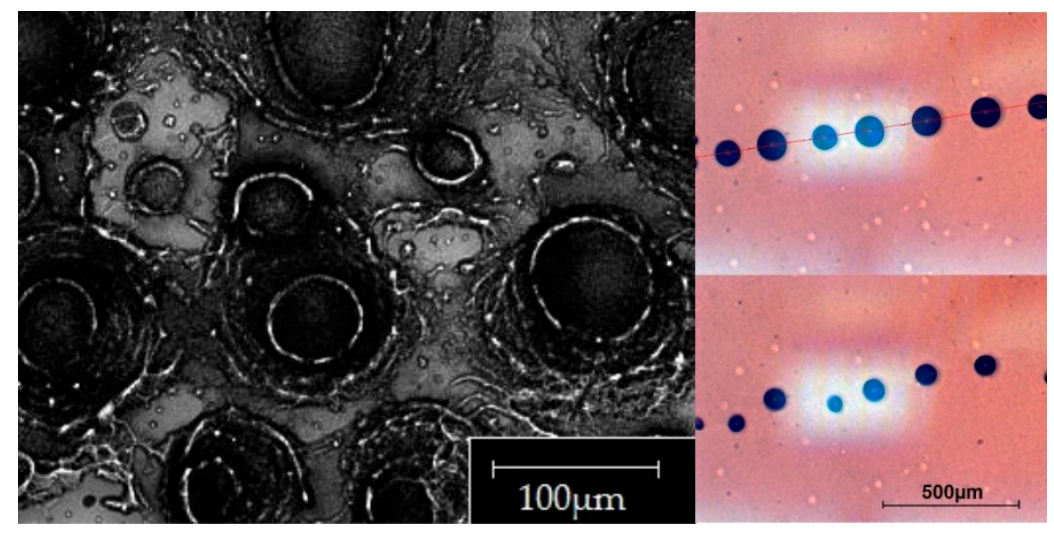

Figure 2. Optical microscope image taken at $20 \times$ magnification of the microdrop deposition of multiple $180 \mathrm{pl}$ droplets with the ink solution deposed on the kapton substrate (left). On the right two microscope images taken at $5 \times$ magnification of a line of distilled water droplets: one at the beginning of the evaporation (top right) and the second after $10 \mathrm{~s}$ (bottom right).

Closer drops would determine a higher relative humidity in the air layers, which surround the drops selves, slowing the evaporation process. At variance, depositing drops at larger distance will limit the amount of deposited liquid actually reducing the integrated efficiency of this process. To further increase the evaporation rate, experiments conducted heating the substrate were also carried out. As expected, as temperature increases, the evaporation rate increases exponentially, with exponential value of the temperature of $\sim 2.5$. For example, at $80{ }^{\circ} \mathrm{C}$, the evaporation rate 
increases about 30 times [9]. However, it is worth noting that increasing temperature could not be always feasible, since increasing the temperatures may affect the chemical stability of the samples. To avoid the problem, thermal radiation focused on droplets may be considered. By selecting specific wavelengths or frequencies, it would be possible to selectively increase the temperature of the solvent and not the one of the PM contained in the solution. Another parameter, which could be modified to fasten the deposition, is the speed of the two motorized stages. Our experiments were all conducted at the maximum allowed speed (i.e., $13 \mathrm{~mm} \cdot \mathrm{s}^{-1}$ ), but faster motorized stages exist that would cover the same area within a shorter time, i.e., just reducing the deposition time between two consecutive drops.

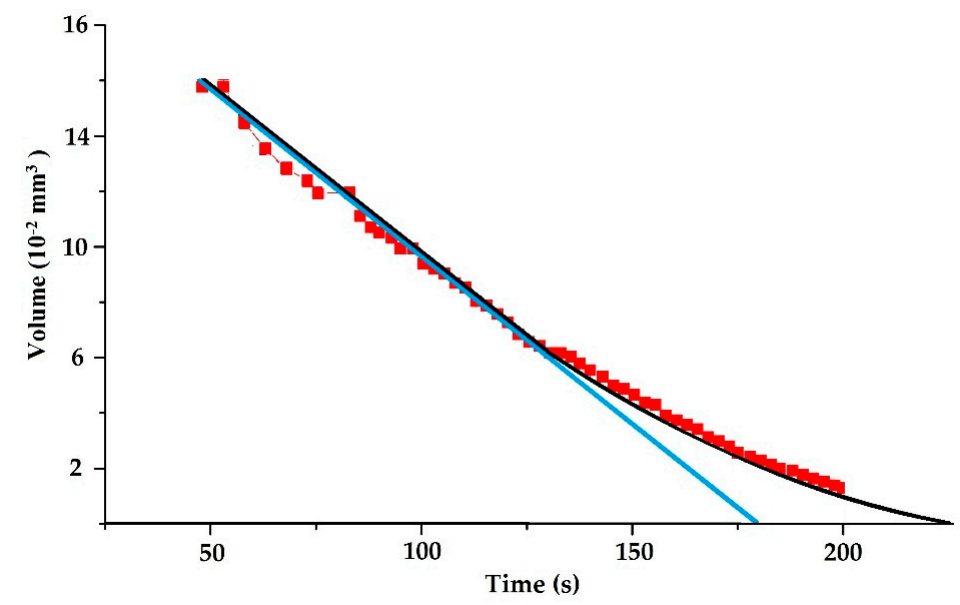

Figure 3. Comparison among the droplet mass behavior for the evaporation at "constant contact area" (blue line), at "constant contact angle" (black line), and experimental data (red squares) of a droplet of a bi-distilled water solution with an initial diameter of $1 \mathrm{~mm}$, at room temperature.

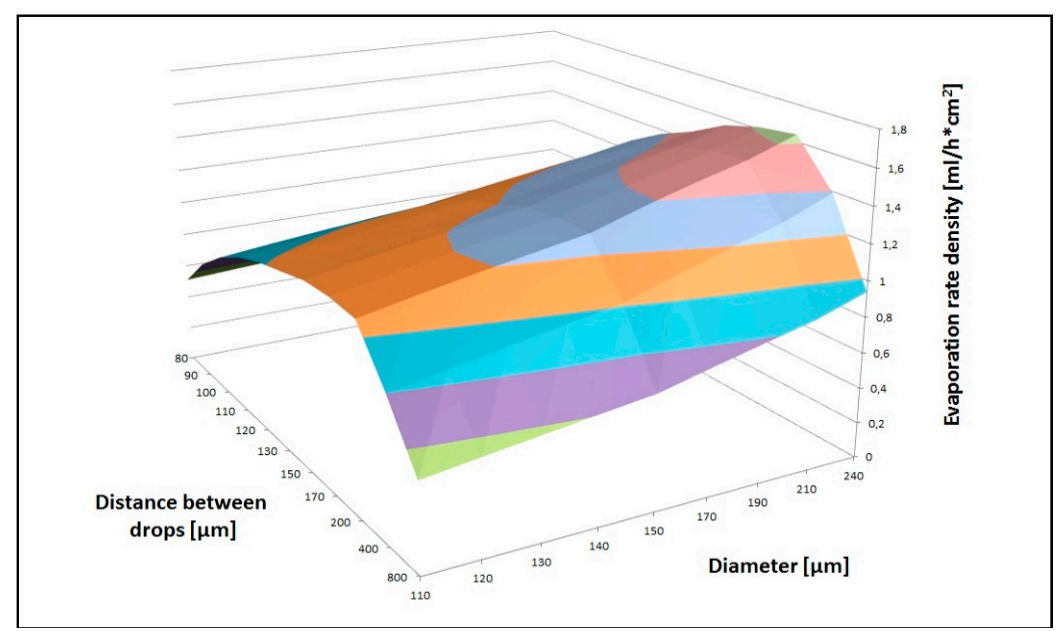

Figure 4. This graph shows the evaporation rate density (volume evaporated per hour and area) at $20^{\circ} \mathrm{C}$ vs. drop diameter and distance between two consecutive drops. Here, the speed has also been considered, i.e., the time associated with the motion of the translational stages used to map with droplets the area to cover.

To better understand the deposition pattern of the PM contained in a liquid solution deposited with a Microdrop dispenser, we prepared a suspension consisting in high purity water (MilliQ technology) and a given amount $(50 \mu \mathrm{g} / \mathrm{mL})$ of a reference material: the NIST standard 2709a. The latter is a well-known soil reference material with a well-characterized composition [11] and grain size distribution. To avoid the clog of the Microdrop nozzle, we selected a reference material 
whose particles didn't exceed $30 \mu \mathrm{m}$, i.e., about the half of the aperture of the nozzle orifice, as shown in Figure 5.

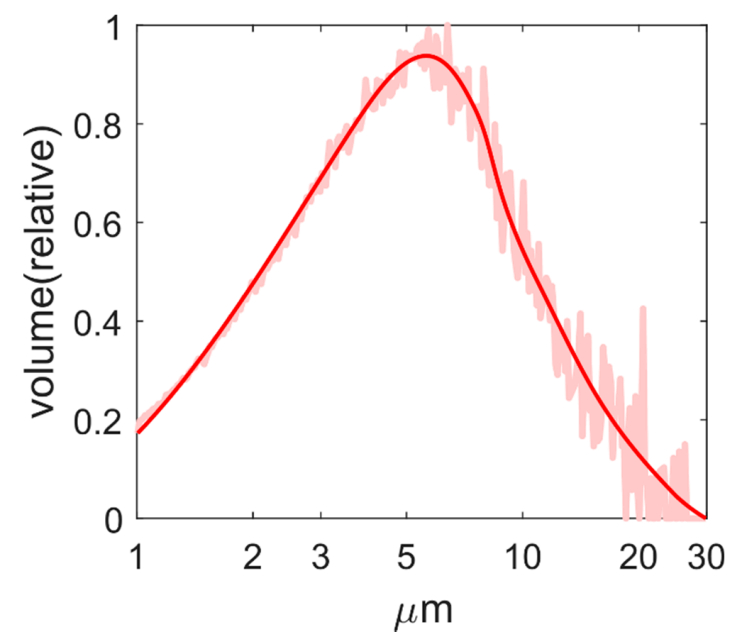

Figure 5. The particle size distribution of the NIST standard reference soil material 2709a. Data were obtained through the Coulter counter technique. Details can be found in [12].

Since the standard contains $\mathrm{Fe}$, it was possible to use the X-ray fluorescence (XRF) technique to investigate its spatial distribution after deposition. In Figure 6, we compare two XRF profiles collected on two deposited samples, measured along a straight line, with a spatial resolution limited by the spot size of the beam $(\sim 200 \mu \mathrm{m})$. The red curve is the profile relative to the deposition of a single large drop of $\sim 1 \mathrm{~mL}$ volume of the NIST solution. The black curve refers to a deposition obtained with the microdrop, made by multiple patterns of $240 \mu \mathrm{m}$ diameter droplets with $170 \mu \mathrm{m}$ between them. The same total volume and the same solution were considered. The two profiles show significant differences and it is remarkable that the homogeneous deposition was obtained with the latter technique, despite the reduced amount of solution. Indeed, the large drop shows density fluctuations from $50 \%$ to $80 \%$ of the fluorescence intensity, in particular at the edge due to the coffee-stain effect [1]. At variance, the variability observed along the microdrop profile is around $10 \%$, and the deposited soil is uniformly distributed.

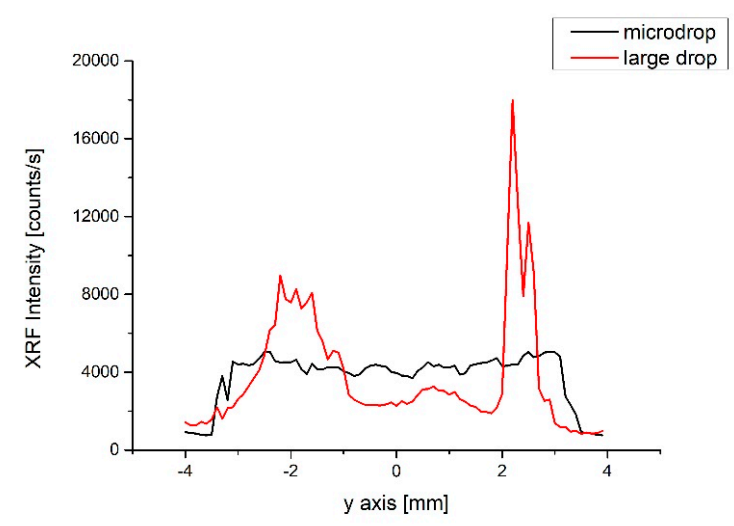

Figure 6. Comparison of two representative X-ray fluorescence profiles at the Fe K-edge: microdrop $5 \times 7 \mathrm{~mm}^{2}$ pattern with drops of $2.9 \mu \mathrm{L}(240 \mu \mathrm{m}$ diameter-black line $)$ and a single large drop deposition (red line). Both samples have been obtained with a total volume deposition of $1 \pm 0.1 \mathrm{~mL}$ of a NIST solution $50 \mu \mathrm{g} / \mathrm{mL}$ concentration on a kapton film. 
Figure 6 compares a microdrop deposition, which clearly shows the homogeneity and the overall quality of the samples prepared with the microdrop increase. The only disadvantage of this method is the deposition time. Indeed, as it can be inferred from Figure 4, the optimal configuration of this micro-deposition allows us to deposit and to evaporate liquid samples with a rate of less than $1 \mathrm{~mL}$ per hour. A simple and alternative way to reduce the deposition time can be obtained by combining the microdrop deposition with a simultaneous filtering technique. In this way, the droplet is deposited onto a wettable filter, and the liquid is pumped out by a vacuum pump.

In Figure 7 (left), the $2 \times 5 \mathrm{~mm}^{2}$ image shows a portion of the original $4 \times 4 \mathrm{~mm}^{2}$ microdrop deposition area of $1 \mathrm{~mL}$ NIST solution $(50 \mu \mathrm{g} / \mathrm{mL}$ concentration) on a polycarbonate Nucleopore $\bigodot$ membrane filter (pore size $0.45 \mu \mathrm{m}$ and filter area diameter $\sim 20 \mathrm{~mm}$ ), which is a hydrophilic substrate. This type of substrate absorbs the liquid solution, and the liquid is continuously pumped out through the filter. The color map was obtained collecting the XRF signal at the Fe K-edge. Colors highlight an area of approximately $2 \times 3 \mathrm{~mm}^{2}$ where a controlled distribution is achieved. The internal variability is probably also related to the spatial pattern of the pores characterizing the filtration membrane.
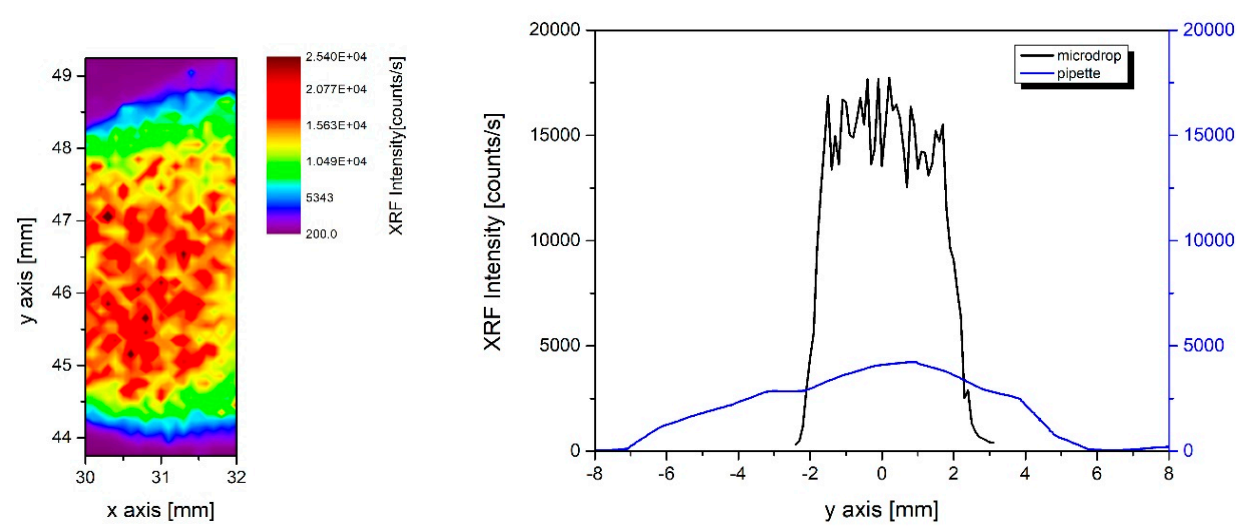

Figure 7. Image of the X-ray Fluorescence at the Fe K-edge of the microdrop deposition area on the filter (left); comparison of two XRF profiles (right): the microdrop deposition (black) and the pipette deposition (blue) both achieved with a simultaneous vacuum filtration technique.

In Figure 7 (right), one microdrop deposition profile of the image on the left is compared with an analogous profile of the deposition of the same solution using a micro-pipette on the same type of filter. From the analysis of the data, we may point out that by using the microdrop deposition, 80\% of the material is distributed quite homogeneously (with a fluorescence intensity fluctuation of $\sim 15 \%$ ) inside an area of $\sim 8 \mathrm{~mm}^{2}$. Using the pipette deposition, the same amount of material is deposited within an area six times larger $\left(\sim 50 \mathrm{~mm}^{2}\right)$. A simple evaluation points out that the microdrop filtration procedure covers an area 6.25 times smaller than the area wetted with the pipette, but the intensity of the fluorescence signal, proportional to the deposed material is four times higher. In this case, the total gain we achieved is $\sim 25$.

\section{Conclusions}

The microdrop technology is suitable to prepare homogeneous deposition of particulate and granular matter samples. In order to establish a reliable procedure, reducing also the deposition time, it is necessary to measure accurately the evaporation rate of the pattern depositions. We show here how to optimize such parameters, increasing the $\mathrm{S} / \mathrm{N}$ ratio and the deposition homogeneity, reducing at the same time the sample consumption. Indeed, $\mathrm{S} / \mathrm{N}$ ratio is related to the XRF signal; increasing the density and the homogeneity of the deposition, the $\mathrm{S} / \mathrm{N}$ ratio of the XRF signal that outlines the distribution of the material in the sample improves. In terms of deposition uniformity, with the microdrop technique, the $\mathrm{S} / \mathrm{N}$ ratio may improve $\sim 4$ times with respect to a classical filtration method. At the same time, it is possible to deposit and evaporate a solution onto any substrate and 
5 times more homogeneously than a large drop evaporation. These features make this technique effective and competitive with respect to many and diverse applications. Among the many, we cite the characterization of aerosols for pollution monitoring purposes, studies of metallic contaminants in polluted water or the investigation of biological diluted materials at ultra-trace concentrations. Besides homogeneity, another important feature of the method proposed here, is the possibility to concentrate in a very reduced area the material contained in an extremely diluted solution or suspension. This makes it possible to consider impurities whose concentrations span from few ppb to hundreds ppm. As an example, the method is suited for the characterization of the inorganic insoluble particulate matter contained in ice core samples for paleoclimatic reconstructions. Ice core dust samples prepared with a microdrop device could be successfully used for both X-rays absorption and X-ray fluorescence measurements $[13,14]$ and under particular experimental conditions also for XRD experiments [15]. Certainly, the microdrop technique has to be improved for applications where beams with spot size well below one micron will be available, as to ultra low brilliance synchrotron radiation facilities and/or Free Electron Lasers. However, due to the low frequency rate of the existing Free Electron Lasers $(10-100 \mathrm{~Hz})$, the technique could already be useful to control the deposition of small samples such as small crystals, clusters, or organic systems delivered to the beam inside droplets.

Author Contributions: S.M., G.C., V.M. and A.M. conceived and designed the experiment; D.H. and S.M. built the experimental setup; S.M. and G.C. performed the experiments and analyzed data; G.B., B.D. and A.D. provided materials and contributed to the data analysis; S.M., G.C., and A.M. wrote the paper. All authors have read and approved the final manuscript.

Funding: This research has been performed in cooperation among INFN-Laboratori Nazionali di Frascati, Milano Bicocca University and the Diamond Light Source facility. The development of the microdrop instrumentation is a project managed by A.M. with the support of DARST-Department of the Presidenza del Consiglio dei Ministri, which is gratefully acknowledged. Part of the preparation was done in the EuroCold Laboratory at University of Milano Bicocca, founded by Italian National Science Foundation NextData Project. Part of this research was developed at Diamond, the UK national synchrotron radiation facility. One of us (S.M.) acknowledges the Roma Tre University for financial support during his stage at Diamond.

Conflicts of Interest: The authors declare no conflict of interest.

\section{References}

1. Innocenzi, P.; Malfatti, L.; Piccinini, M.; Grosso, D.; Marcelli, A. Stain Effects Studied by Time-Resolved Infrared Imaging. Anal. Chem. 2009, 81, 551-556. [CrossRef] [PubMed]

2. Picknett, R.G.; Bexon, R. The evaporation of sessile or pendant drops in still air. J. Colloid Interface Sci. 1977, 61, 336-350. [CrossRef]

3. Hu, H.; Larson, R.G. Evaporation of a sessile droplet on a substrate. J. Phys. Chem. B 2002, 106, $1334-1344$. [CrossRef]

4. Girard, F.; Antoni, M.; Faure, S.; Steinchen, A. Evaporation and Marangoni Driven Convection in Small Heated Water Droplets. Langmuir 2006, 22, 11085-11091. [CrossRef] [PubMed]

5. Peschel, B.U.; Fittschen, U.E.A.; Pepponi, G.; Jokubonis, C.; Streli, C.; Wobrauschek, P.; Falkenberg, G.; Broekaert, J.A.C. Direct analysis of $\mathrm{Al}_{2} \mathrm{O}_{3}$ powders by total reflection X-ray fluorescence spectrometry. Anal. Bioanal. Chem. 2005, 382, 1958-1964. [CrossRef] [PubMed]

6. Fittschen, U.E.A.; Bings, N.H.; Hauschild, S.; Förster, S.; Kiera, A.F.; Karavani, E.; Frömsdorf, A.; Thiele, J.; Falkenberg, G. Characteristics of picoliter droplet dried residues as standards for direct analysis techniques. Anal. Chem. 2008, 80, 1967-1977. [CrossRef] [PubMed]

7. Sparks, C.M.; Fittschen, U.E.A.; Havrilla, G.J. Picoliter solution deposition for total reflection X-ray fluorescence analysis of semiconductor samples. Spectrochim. Acta Part B 2010, 65, 805-811. [CrossRef]

8. Meyer, W. Mikroverklebungen aus dem Tintenstrahldrucker PLUS; Eugen, G., Ed.; Leuze Verlag: Norderstedt, Germany, 2013; pp. 593-598. (In German)

9. Macis, S. Preparation and Characterization of Ultra-Diluted Samples via Micro-Deposition of Droplets. Master's Thesis, Roma Tre University, Roma, Italy, 2014. 
10. Dent, J.; Cibin, G.; Ramos, S.; Parry, S.A.; Gianolio, D.; Smith, A.D.; Scott, S.M.; Varandas, L.; Patel, S.; Pearson, M.R.; et al. Performance of B18, the Core EXAFS Bending Magnet beamline at Diamond. J. Phys. Conf. Ser. 2013, 430, 012023. [CrossRef]

11. Mackey, E.A.; Christopher, S.J.; Lindstrom, R.M.; Long, S.E.; Marlow, A.F.; Murphy, K.E.; Paul, R.L.; Popelka-Filcoff, R.S.; Rabb, S.A.; Sieber, J.R.; et al. Nebelsick, Certification of Three NIST Renewal Soil Standard Reference Materials for Element Content: SRM 2709a San Joaquin Soil, SRM 2710a Montana Soil I, and SRM 2711a Montana Soil II; NIST Special Publication 260-172; National Institute of Standards and Technology: Gaithersburg, MD, USA, 2010; p. 39.

12. Ruth, U.; Barbante, C.; Bigler, M.; Delmonte, B.; Fischer, H.; Gabrielli, P.; Gaspari, V.; Kaufmann, P.; Lambert, F.; Maggi, V.; et al. Proxies and Measurement Techniques for Mineral Dust in Antarctic Ice Cores. Environ. Sci. Technol. 2008, 42, 5675-5681. [CrossRef] [PubMed]

13. Marcelli, A.; Cibin, G.; Hampai, D.; Giannone, F.; Sala, M.; Pignotti, S.; Maggi, V.; Marino, F. XANES characterization of deep ice core insoluble dust in the ppb range. J. Anal. At. Spectrom. 2012, 27, 33-37. [CrossRef]

14. Marcelli, A.; Maggi, V. Aerosols in Snow and Ice. Markers of Environmental Pollution and Climatic Changes: European and Asian Perspectives; Superstripes Press: Rome, Italy, 2017; ISBN 9788866830771.

15. D'Elia, A.; Cibin, G.; Robbins, P.E.; Maggi, V.; Marcelli, A. Design and characterization of a mapping device optimized to collect XRD patterns from highly inhomogeneous and low density powder samples. Nucl. Instrum. Methods Phys. Res. B 2017, 411, 22-28. [CrossRef]

(C) 2018 by the authors. Licensee MDPI, Basel, Switzerland. This article is an open access article distributed under the terms and conditions of the Creative Commons Attribution (CC BY) license (http:/ / creativecommons.org/licenses/by/4.0/). 